TITLE:

\title{
Size-dependent effect of gold nanospheres on the acoustic pressure pulses from laser- irradiated suspensions
}

\section{AUTHOR(S):}

Fukasawa, Tomonori; Shinto, Hiroyuki; Aoki, Hiroyuki; Ito, Shinzaburo; Ohshima, Masahiro

\section{CITATION:}

Fukasawa, Tomonori ... [et al]. Size-dependent effect of gold nanospheres on the acoustic pressure pulses from laser-irradiated suspensions. Advanced Powder Technology 2014, 25(2): 733-738

\section{ISSUE DATE:}

2014-03

URL:

http://hdl.handle.net/2433/187109

\section{RIGHT:}

(c) 2013 The Society of Powder Technology Japan. Published by Elsevier B.V.; This is not the published version. Please cite only the published version.; この論文は出版社版でありません。引用の際には出版社版を ご確認ご利用ください。 


\section{Size-dependent effect of gold nanospheres on the}

\section{acoustic pressure pulses from laser-irradiated}

\section{suspensions}

Tomonori Fukasawa ${ }^{1}$, Hiroyuki Shinto ${ }^{1, *}$, Hiroyuki Aoki ${ }^{2,3}$, Shinzaburo Ito ${ }^{2,3}$, and Masahiro Ohshima $^{1}$

${ }^{1}$ Department of Chemical Engineering, ${ }^{2}$ Department of Polymer Chemistry, and ${ }^{3}$ Advanced Biomedical Engineering Research Unit, Kyoto University, Katsura, Nishikyo-ku, Kyoto 615-8510, Japan

*Author to whom correspondence should be addressed.

Phone: +81-75-383-2671, Fax: +81-75-383-2651, e-mail: shinto@ cheme.kyoto-u.ac.jp 


\begin{abstract}
We carried out the experimental measurements of photoacoustic responses, where the suspensions of spherical gold nanoparticles (NPs) of different diameters $(20,40$, and $50 \mathrm{~nm})$ in water at different concentrations and different temperatures $\left(4\right.$ and $20^{\circ} \mathrm{C}$ ) were irradiated by 0.8 -ns laser pulses. In the case of $20{ }^{\circ} \mathrm{C}$, the values of photoacoustic signals normalized by the light absorbance of the NP suspension decreased with increasing the NP size. The photoacoustic signals at $4{ }^{\circ} \mathrm{C}$ were significantly reduced compared with those at $20{ }^{\circ} \mathrm{C}$. These experimental results are in fair agreement with the estimations from our phenomenological model, where the acoustic pressure pulse is represented by a sum of two contributions from the NPs and the surrounding liquid medium.
\end{abstract}

Keywords:

Photoacoustic pressure; Size-dependent effect; Metal nanoparticles; Photoacoustic imaging; Contrast agents; Nanosecond laser pulse. 


\section{Introduction}

In recent years, bioimaging techniques for clinical diagnostics have received great attention and various techniques have been developed to improve detection sensitivity and treatment outcome in patients. One example is photoacoustic imaging (PAI), which is a nonionizing and noninvasive imaging modality based on the photoacoustic effect: the irradiated light is absorbed by a target to be converted into a thermoacoustic wave that is detected by an acoustic transducer $[1,2]$. The contrast in PAI counts on the optical-to-acoustic conversion (optoacoustic) efficiency, and can be exogenously improved by use of nanoparticles (NPs) as contrast agents [2-4]. With recent growing advancement in nanotechnology, a large variety of NPs have been developed for the PAI contrast agents $[5,6]$.

Henceforth, for the sake of clarity, let us consider a simple system, that is, a weakly lightabsorbing liquid dispersed with strongly absorbing NPs, where the amplitude of the photoacoustic signal should depend largely on the transfer of heat between two materials of the NPs and host liquid. At low laser fluence, the acoustic signal results from the thermoacoustic response of the heated host liquid as well as that of the heated NPs. If the laser fluence reaches a threshold, the temperature of the NPs can exceed the boiling temperature of the host liquid. Subsequently, a vapor layer appears on the surface of every NP to undergo rapid expansion, thus giving rise to an effective photoacoustic signal. This cavitation-induced enhancement of the photoacoustic signal at high laser fluence has been investigated in several experimental and theoretical studies [7-17], the principal focus of which was placed on direct observations and theoretical descriptions of bubble formation around heated particles and the resultant enhanced amplitude of the acoustic signal. On the other hand, very few studies have reported experimental results and physical modeling in the case of low-level laser irradiation that help us to better understand the fundamentals of the acoustic signal resulting from the thermoacoustic response of the heated NPs and heated host liquid in the absence of cavitation, except for the following studies.

In a recent study, Chen et al. [18] have demonstrated that the acoustic signal generated from laserirradiated suspensions of spherical 26-nm gold NPs is influenced by the temperature- and material- 
dependent properties of the host liquids (i.e., water, toluene, and silicone oil), and that the photoacoustic signal is dominated by the surrounding liquid rather than the NPs although the light absorbance of the former is still weaker than that of the latter. Only a few studies [18-22] have reported physical modeling of the acoustic signal produced from such a heated inhomogeneous solution including NPs (other than the particles of the larger sizes ranging from micrometers to millimeters) in the case of low-level laser irradiation. Nevertheless, the effects of NPs on the photoacoustic phenomena are not understood well and remain to be explored experimentally and theoretically from a fundamental point of view.

Main focus of the present study is placed on how the thermoacoustic responses from a laserirradiated suspension of NPs are influenced by the size of the NPs. We report the experimental measurements of the photoacoustic signals, where the suspensions of spherical gold NPs of different diameters in water at different concentrations and temperatures are irradiated by nanosecond laser pulses at low laser fluence. The results from the experiments are compared with those from our phenomenological model that is developed from our previous study [19].

\section{Experimental methods}

\subsection{Gold nanospheres}

The aqueous suspensions of spherical gold NPs of different diameters $\left(d_{\mathrm{p}}=20,40\right.$, and $50 \mathrm{~nm}$ at the number concentrations of $7.0 \times 10^{11}, 9.0 \times 10^{10}$, and $4.5 \times 10^{10}$ particles $/ \mathrm{mL}$, respectively) were purchased from British BioCell International (Cardiff, UK). The concentrated suspensions were prepared in clean test tubes by solvent evaporation at $60{ }^{\circ} \mathrm{C}$ [19]. Before and after this concentration process, no significant change was found in the profile of the absorption spectra of the gold NP suspensions, indicating that the concentration process hardly affected the morphology and the monodispersity of the gold NPs. 


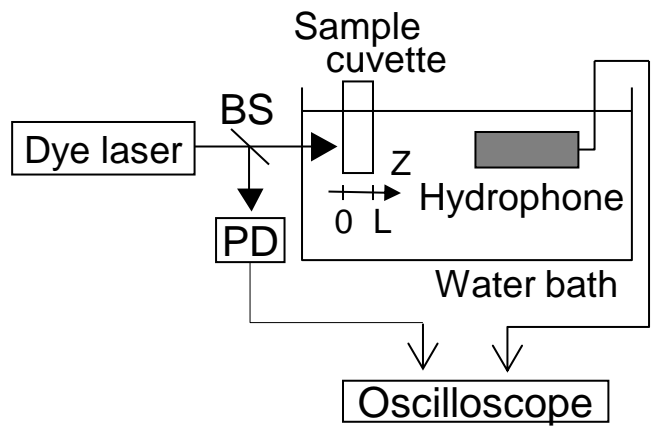

Fig. 1. Illustration of the experimental setup. BS: beam splitter, PD: photo-diode.

\subsection{Setup for photoacoustic signal detection}

Figure 1 depicts our experimental setup for photoacoustic signal detection, which is the same as employed in our previous study [19]. A sample cuvette was immersed in a temperature-controlled water bath and illuminated by a pulsed beam from a dye laser pumped by a nitrogen laser (OB401/OB-4300; Optical Building Blocks, Birmingham, NJ, USA), where the pulse width and repetition rate were $0.8 \mathrm{~ns}\left(=t_{\mathrm{L}}\right)$ and $5 \mathrm{~Hz}$, respectively. The wavelength of this laser was set at 522 $\mathrm{nm}$, where the energy was $I_{0} \equiv 107 \mu \mathrm{J} /$ pulse. The cross section of the specific region irradiated by the laser pulse was $s_{\mathrm{L}} \equiv 0.524 \mathrm{~mm}^{2}$ and the laser fluence was $F_{0} \equiv I_{0} / \mathrm{s}_{\mathrm{L}}=204 \mathrm{~J} /\left(\mathrm{m}^{2}\right.$ pulse $)$. The acoustic signal from the laser-irradiated gold suspension was detected by a membrane-type hydrophone (H9C-036; Toray Engineering, Japan) with a measurable frequency range of 0.5-10 MHz. The distance between the hydrophone and the center of the sample cuvette was $\approx 38 \mathrm{~mm}$. The output signal from the hydrophone was recorded by an oscilloscope (TDS-2012; Tektronix, Beaverton, OR, USA) through an amplifier (Model 5682; Olympus NDT, Waltham, MA, USA). The split laser beam was detected by a photo-diode (DET10A; Thorlabs, Newton, NJ, USA) and fed to the oscilloscope as the trigger signal. The measurements were performed at the suspension temperatures of 4 and $20{ }^{\circ} \mathrm{C}$, using the cuvettes of light pass length of $L=0.92 \mathrm{~mm}$. The results were obtained after averaging over 128 signals from every sample at each temperature. After that, the absorption spectra of these laser irradiated suspensions were measured. Before and after the laser irradiation, no significant change was observed in the profile of the absorption spectra of the suspensions, implying that the laser irradiation hardly caused the fragmentation/aggregation of the 
gold NPs.

\section{Theoretical descriptions}

\subsection{Thermodynamic relation for a heated monodispersed suspension containing particles}

Let us consider a heated monodispersed suspension containing particles at the number density of $n_{\mathrm{p}}$. Supposed that every particle has the thermal energy therein $\left(e_{\mathrm{p}}\right)$ and the surrounding medium has the energy resulting from the heat conduction from the particle thereto $\left(e_{\mathrm{m}}\right)$ immediately after the laser irradiation with a nanosecond pulse, where $e_{\text {tot }}\left(\equiv e_{\mathrm{p}}+e_{\mathrm{m}}\right)$ represents the total energy per particle. The physical properties of the particle and medium are defined as follows: $c_{\mathrm{i}}$, the specific heat capacity; $\rho_{\mathrm{i}}$, the mass density; $\kappa_{\mathrm{i}}$, the isothermal compressibility; $\beta_{\mathrm{i}}$, the thermal coefficient of volume expansion, where the subscript i denotes the particle $(\mathrm{p})$ or the medium $(\mathrm{m})$. Hereafter, we assume that the physical properties of the particle and medium (i.e., $c_{\mathrm{i}}, \rho_{\mathrm{i}}, \kappa_{\mathrm{i}}$, and $\beta_{\mathrm{i}}$ ) are constant at the initial temperature.

Starting from the thermodynamic relation $(\Delta V / V=-\kappa \Delta P+\beta \Delta T$, where $\Delta V, \Delta P$, and $\Delta T$ denote the volume, pressure, and temperature changes of a system of interest, respectively), we have derived the local pressure rise $p_{\text {tot }}$ of an inhomogeneous liquid containing particles upon short-pulsed laser irradiation and two contributions from the particles $\left(p_{\mathrm{p}}\right)$ and the medium $\left(p_{\mathrm{m}}\right)$, as elaborated in Ref. [19]:

$$
\begin{aligned}
& p_{\text {tot }}=n_{\mathrm{p}} e_{\mathrm{tot}} \cdot\left[\left(\kappa_{\mathrm{p}} \Gamma_{\mathrm{p}} / \kappa_{\mathrm{m}}\right) \cdot x+\Gamma_{\mathrm{m}} \cdot(1-x)\right]=p_{\mathrm{p}}+p_{\mathrm{m}} \\
& p_{\mathrm{p}} \equiv\left(\kappa_{\mathrm{p}} \Gamma_{\mathrm{p}} / \kappa_{\mathrm{m}}\right) \cdot\left(n_{\mathrm{p}} e_{\mathrm{tot}}\right) x \\
& p_{\mathrm{m}} \equiv \Gamma_{\mathrm{m}} \cdot\left(n_{\mathrm{p}} e_{\mathrm{tot}}\right)(1-x)
\end{aligned}
$$

with

$$
\begin{aligned}
& \Gamma_{\mathrm{i}} \equiv \beta_{\mathrm{i}} / \kappa_{\mathrm{i}} c_{\mathrm{i}} \rho_{\mathrm{i}} \\
& x \equiv e_{\mathrm{p}} / e_{\mathrm{tot}}, \quad 1-x \equiv e_{\mathrm{m}} / e_{\mathrm{tot}}
\end{aligned}
$$

where $\Gamma_{\mathrm{i}}$ denotes the Grüneisen parameter for the particle $(\mathrm{i}=\mathrm{p})$ or medium $(\mathrm{i}=\mathrm{m})$ and $n_{\mathrm{p}} e_{\mathrm{tot}}$ 
represents the volumetric optical absorption by the particle suspension. As will be expected from Fig. 5 , the limiting of $x\left(\equiv e_{\mathrm{p}} / e_{\mathrm{tot}}\right) \rightarrow 0$ is equivalent to that of (particle size) $\rightarrow 0$, where the particles are considered to be dissolved as solute molecules in the medium at a certain concentration. In this case, Eq. (1a) reduces to $p_{\text {tot }}=\left(n_{\mathrm{p}} e_{\mathrm{tot}}\right) \Gamma_{\mathrm{m}}$, which is a popular form of the acoustic pressure [1, 23]. It is worth noting that Eq. (1) is consistent with the idea given in Ref. [18]: If the pressure is measured in the medium far from the particle, the contributions from the solid particle and the liquid environment can be separated by setting the liquid and the solid volume expansion coefficients, respectively, to zero. Consequently, we can estimate the pressure rise $p_{\text {tot }}$ and two contributions, $p_{\mathrm{p}}$ and $p_{\mathrm{m}}$, from Eq. (1), if the thermal energies per particle, $e_{\mathrm{tot}}, e_{\mathrm{p}}$, and $e_{\mathrm{m}}$, are obtained.

3.2. Heat production by a laser-irradiated metal nanosphere and the heat transfer into its surrounding liquid

Let us consider a single spherical particle of diameter $d_{\mathrm{p}}\left(=2 r_{\mathrm{p}}\right)$ suspended in an infinite liquid medium irradiated by a laser pulse, in order to calculate the thermal energies per particle, $e_{\mathrm{tot}}, e_{\mathrm{p}}$, and $e_{\mathrm{m}}$. It is assumed that the energy of the laser pulse absorbed by the particle is completely utilized to heat the particle and surrounding medium. The heat transfer equations for the single particle and surrounding medium with spherical symmetry are given by

$c_{\mathrm{p}} \rho_{\mathrm{p}} \frac{\partial T_{\mathrm{p}}(r, t)}{\partial t}=\frac{1}{r^{2}} \frac{\partial}{\partial r}\left[\left.r^{2} \cdot k_{\mathrm{p}} \frac{\partial T_{\mathrm{p}}(r, t)}{\partial r}\right|_{]}+S(r, t), \quad r \leq r_{\mathrm{p}}\right.$

$c_{\mathrm{m}} \rho_{\mathrm{m}} \frac{\partial T_{\mathrm{m}}(r, t)}{\partial t}=\frac{1}{r^{2}} \frac{\partial}{\partial r}\left[\left.r^{2} \cdot k_{\mathrm{m}} \frac{\partial T_{\mathrm{m}}(r, t)}{\partial r}\right|_{]}, \quad r \geq r_{\mathrm{p}}\right.$

respectively, where $k_{\mathrm{i}}(\mathrm{i}=\mathrm{p}, \mathrm{m})$ denote the thermal conductivity. The radiation energy absorbed by the particle, $S(r, t)$, is assumed to be homogeneously distributed over the particle volume, $v_{\mathrm{p}}$ $\left(=4 \pi r_{\mathrm{p}}{ }^{3} / 3\right)$, following

$S(r, t) \equiv \frac{\sigma_{\text {abs }} q(t)}{v_{\mathrm{p}}}$

where $\sigma_{\mathrm{abs}}$ is the absorption cross-section of the particle. It is noted that $\sigma_{\mathrm{abs}} \propto v_{\mathrm{p}}$ for sufficiently 
small particles (e.g., $d_{\mathrm{p}}<60 \mathrm{~nm}$ for gold particles), whereas $\sigma_{\mathrm{abs}} \propto s_{\mathrm{p}}\left(=4 \pi r_{\mathrm{p}}{ }^{2}\right)$ for sufficiently large particles. The light intensity, $q(t)$, is represented by a rectangular profile with the full width at half maximum $(\mathrm{FWHM}), t_{\mathrm{L}}$, using

$q(t)=\begin{array}{ll}\vdots q_{0}, & 0\lrcorner t\lrcorner t_{\mathrm{L}} \\ \vdots 0, & t \text { ウ } t_{\mathrm{L}}\end{array}$

where the laser fluence is given as $F_{0}=q_{0} t_{\mathrm{L}}$.

If thermal contact resistance exists at the interface between the particle and its surrounding liquid medium, the interface boundary conditions for Eq. (4) are given by

$-\left.k_{\mathrm{p}} \frac{\partial T_{\mathrm{p}}(r, t)}{\partial r}\right|_{r=r_{\mathrm{p}}}=\left.h_{\mathrm{I}}\left[T_{\mathrm{p}}(r, t)-T_{\mathrm{m}}(r, t)\right]\right|_{r=r_{\mathrm{p}}}=-\left.k_{\mathrm{m}} \frac{\partial T_{\mathrm{m}}(r, t)}{\partial r}\right|_{r=r_{\mathrm{p}}}$

where $h_{\mathrm{I}}$ denotes the interface thermal contact conductance, the reciprocal of which is the thermal contact resistance. On the other hand, if there is no interface thermal contact resistance (i.e., $\left.h_{\mathrm{I}} \rightarrow \infty\right)$, Eq. (7) is replaced with the following:

$\left.T_{\mathrm{p}}(r, t)\right|_{r=r_{\mathrm{p}}}=\left.T_{\mathrm{m}}(r, t)\right|_{r=r_{\mathrm{p}}}$

$-\left.k_{\mathrm{p}} \frac{\partial T_{\mathrm{p}}(r, t)}{\partial r}\right|_{r=r_{\mathrm{p}}}=-\left.k_{\mathrm{m}} \frac{\partial T_{\mathrm{m}}(r, t)}{\partial r}\right|_{r=r_{\mathrm{p}}}$

The initial conditions for Eq. (4) are given by

$$
\begin{cases}T_{\mathrm{p}}(r, 0)=T_{\text {init }}, & r \leq r_{\mathrm{p}} \\ T_{\mathrm{m}}(r, 0)=T_{\text {init }}, & r \geq r_{\mathrm{p}}\end{cases}
$$

where $T_{\text {init }}$ is the temperature before laser irradiation.

It should be noted that no thermal expansion of the single particle and surrounding medium is implicitly assumed during/after laser irradiation.

\subsection{Numerical simulations}

Hereafter, we assume that the physical properties of a particle and a medium (i.e., $c_{\mathrm{i}}, \rho_{\mathrm{i}}, \kappa_{\mathrm{i}}, \beta_{\mathrm{i}}, k_{\mathrm{i}}$, and $\left.\sigma_{\mathrm{abs}}\right)$ are the constant values at the temperature before laser irradiation. We considered the 
spherical gold NPs of diameter $d_{\mathrm{p}}=2 r_{\mathrm{p}} \leq 60 \mathrm{~nm}$ suspended in water medium, which were irradiated by a laser beam with a rectangular pulse [FWHM $t_{\mathrm{L}}=0.8 \mathrm{~ns}$ and the laser fluence $F_{0}=204 \mathrm{~J} /\left(\mathrm{m}^{2}\right.$ pulse)]. This simulation system corresponds to the experimental system mentioned in Section 2 . The physical properties of gold and water at 4 and $20^{\circ} \mathrm{C}$ are listed in Table S1. The absorption crosssection $\sigma_{\text {abs }}$ of a spherical gold NP in water at $\lambda=522 \mathrm{~nm}$ was calculated as a function of the particle diameter, following Ref. [24]. We numerically solved Eq. (4) with Eqs. (5), (6), (8), and (9) using a finite volume method with the fully implicit scheme and the tri-diagonal matrix algorithm [25] to obtain the temperature profile of $T(r, t)$ as a function of the radial position $r$ and time $t$. In contrast, we employed the analytical description of $T(r, t)$ in our previous study [19], where the spatial temperature inhomogeneity inside an NP was assumed to be negligibly small compared with the temperature distribution of the surrounding medium.

In the absence of laser attenuation, the total thermal energy produced by a laser-irradiated particle $e_{\text {tot }}(t)$, the thermal energy inside the particle $e_{\mathrm{p}}(t)$, and the thermal energy of its surrounding medium $e_{\mathrm{m}}(t)$ are respectively given as a function of time:

$e_{\mathrm{tot}}(t)=\sigma_{\mathrm{abs}} \int_{0}^{t} q(\tau) \mathrm{d} \tau$

$e_{\mathrm{p}}(t)=c_{\mathrm{p}} \rho_{\mathrm{p}} \int_{0}^{r_{\mathrm{p}}}\left[T_{\mathrm{p}}(r, t)-T_{\text {init }}\right] 4 \pi r^{2} \mathrm{~d} r$

$e_{\mathrm{m}}(t) \equiv e_{\mathrm{tot}}(t)-e_{\mathrm{p}}(t)$

\subsection{Photoacoustic pressure pulse from particle suspension at different concentrations}

Once the time at an instant of thermal-to-acoustic conversion $\left(t_{\text {conv }}\right)$ is assumed properly, we can straightforward estimate the acoustic pressure pulse in the absence of laser and sound attenuation, using Eq. (1) with the values of $e_{\mathrm{i}}(t)$ (i=tot, $\left.\mathrm{p}, \mathrm{m}\right)$ at $t=t_{\text {conv }}$ that are obtained from Eq. (10) with the numerical solution of $T(r, t)$ for Eq. (4). Following Egerev et al. [11], $t_{\text {conv }}$ was assumed to be the time for the end of laser irradiation, namely $t_{\mathrm{conv}}=t_{\mathrm{L}}(=0.8 \mathrm{~ns})$ in the present study.

The light absorbance $A_{\lambda}$ for the monodispersed suspension of particles in the cuvette of the light pass length of $L$ is defined as 
$A_{\lambda} \equiv-\log _{10}\left[I(L) / I_{0}\right]=n_{\mathrm{p}} \sigma_{\mathrm{ext}} L / \log _{e} 10$

$I(L) \equiv I_{0} \cdot \exp \left(-n_{\mathrm{p}} \sigma_{\mathrm{ext}} L\right)$

where $\sigma_{\text {ext }}$ is the extinction cross-section of the particle and $I_{0} \equiv s_{\mathrm{L}} F_{0}$. If the particles are sufficiently small, the extinction cross-section is approximated as $\sigma_{\mathrm{ext}} \approx \sigma_{\mathrm{abs}}$, leading to

$A_{\lambda} \approx n_{\mathrm{p}} \sigma_{\mathrm{abs}} L / \log _{e} 10=n_{\mathrm{p}} e_{0} L /\left(F_{0} \log _{e} 10\right)$

where $e_{0} \equiv \sigma_{\text {abs }} F_{0}$ denotes the optical power deposition per particle. Using Eqs. (1) and (13) with $e_{\text {tot }}$ $=e_{0}$, the photoacoustic pressure pulse in the absence of laser and sound attenuation is given by

$p_{\mathrm{tot}} \approx\left[\left(F_{0} \log _{e} 10\right) / L\right] \times\left[\left(\kappa_{\mathrm{p}} \Gamma_{\mathrm{p}} / \kappa_{\mathrm{m}}-\Gamma_{\mathrm{m}}\right) \cdot x+\Gamma_{\mathrm{m}}\right] \cdot A_{\lambda}$

Equation (14) is useful for analyzing the photoacoustic signals observed from the particle suspensions of different particle sizes and concentrations, as will be shown in Section 4.3.

\section{Results and discussion}

\subsection{Observation of photoacoustic signal amplitudes}

Figure 2 displays the signal amplitude observed from a laser-irradiated aqueous suspension of 50nm gold NPs at $20^{\circ} \mathrm{C}$, as a function of time. Two isolated spikes were observed as indicated by the arrows in Fig. 2: the positive-peak signal was first observed and followed by the negative-peak signal after the time period of $0.62 \mu \mathrm{s}$. The time lag between these first and second peak signals exactly coincides with the value of $L / C_{0, \mathrm{~m}}=0.62 \mu \mathrm{s}$ for the sample cuvette of $L=0.92 \mathrm{~mm}$, where the speed of sound wave in water, $C_{0, \mathrm{~m}}=1481 \mathrm{~m} / \mathrm{s}$, was used. These results indicate that the peaks of the first and second signals in Fig. 2 correspond to the plane waves generated at the inner walls of the cuvette, $Z=L$ and 0 , respectively (see also Fig. 1). Thus, the transducer output was not a pressure pulse signature, but its derivative, where the profile of photoacoustic signal observed by our experimental setup reflected the shape of a laser-irradiated domain of particle suspension in a cuvette rather than the morphology of individual particles [19]. The peak signals at $Z=0$ were used as a measure of the photoacoustic responses to avoid the complication from laser attenuation in the 


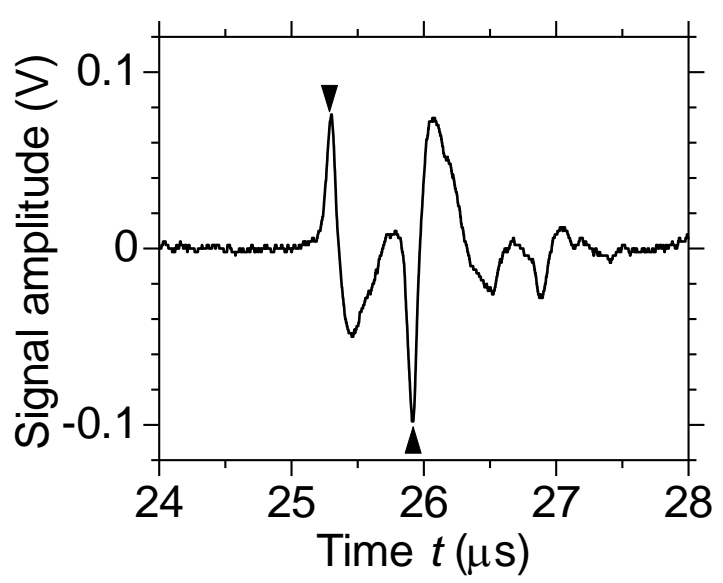

Fig. 2. Time variation of acoustic signal amplitudes observed from a laser-irradiated aqueous suspension of $50-\mathrm{nm}$ gold NPs at $20^{\circ} \mathrm{C}$. The light absorbance of this suspension was $A_{522}=0.33$, which is equivalent to the number concentration of $n_{\mathrm{p}}=1.1 \times 10^{11}$ particles $/ \mathrm{mL}$. The arrows indicate two isolated spikes observed.

present study, whereas those at $Z=L$ were affected by the laser attenuation as experimentally/theoretically demonstrated in Ref. [19]. The detailed results of the photoacoustic signals observed are summarized in Fig. S1-S3.

Figure 3 shows the peak values of the photoacoustic signals from suspensions of gold NPs for different diameters of $d_{\mathrm{p}}=20,40$, and $50 \mathrm{~nm}$ at different temperatures of 4 and $20{ }^{\circ} \mathrm{C}$, as a function of the absorbance of the suspension $A_{522}$, which was measured using the cuvettes of $L=0.92 \mathrm{~mm}$ and represented by Eq. (11). The peak signal for the suspension of each NP size at $20{ }^{\circ} \mathrm{C}$ linearly increased with the absorbance in the range of $A_{522}<0.35$. This behavior is in good agreement with the results of Copland et al. [26], where the photoacoustic signal amplitude from 40-nm gold NPs embedded in the gelatin phantom linearly increases with the concentration up to $n_{\mathrm{p}}=1.2 \times 10^{10}$ particles/mL. It is noted that $A_{\lambda}$ is directly proportional to the number concentration of the suspended particles, $n_{\mathrm{p}}$, via Eq. (13), when the suspensions are sufficiently dilute and the particles are sufficiently small. These results are consistent with the theoretical estimation of Eq. (14). Several experimental studies have reported that the photoacoustic pressures from a laser-irradiated water and a laser-irradiated suspension containing particles increase linearly with the laser fluence under 


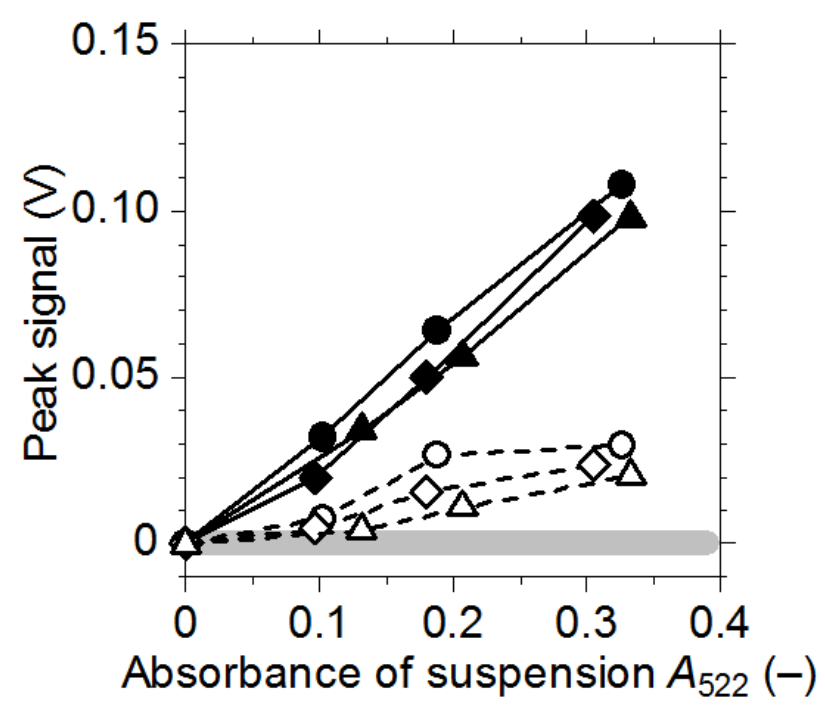

Fig. 3. The peak values of the acoustic signals observed from a laser-irradiated aqueous of 20-nm (circles), 40-nm (diamonds) and 50-nm (triangles) gold NPs, as a function of the absorbance of the suspension $A_{522}$ for different temperatures of $4^{\circ} \mathrm{C}$ (open symbols) and $20^{\circ} \mathrm{C}$ (filled symbols). The dashed and solid lines are the guides for the eyes. The thick, gray-colored line indicates the level of the background noise.

sufficiently low laser fluence $[7,8,15,16,27]$ : this linear increase of the photoacoustic pressure is explained by Eq. (14) as well. If the laser fluence exceeds a threshold, the photoacoustic pressure experiences a sharp nonlinear increase, where the water layer adjacent to heated metal NPs undergoes a phase transition from liquid to vapor [7-17]; this is out of our scope in the present study.

In the case of $4{ }^{\circ} \mathrm{C}$, the peak signals were significantly reduced compared with those at $20{ }^{\circ} \mathrm{C}$. The peak signals at $A_{522} \approx 0.1$ were very close to the level of the background noise, although they slightly increased with the absorbance in the range of $A_{522}>0.15$. Similar behavior has been reported by Chen et al. [18], where the photoacoustic signals for an aqueous suspension of 26-nm gold NPs irradiated by 5 -ns laser pulses becomes negligibly small at $4{ }^{\circ} \mathrm{C}$.

\subsection{Theoretical estimation of temperatures and thermal energies}

As described in Section 3.3, we computed the temperature profile for a spherical gold NP $\left(d_{\mathrm{p}}=20\right.$ or $50 \mathrm{~nm}$ ) and the surrounding water, which were irradiated by the laser pulse. The absorption 


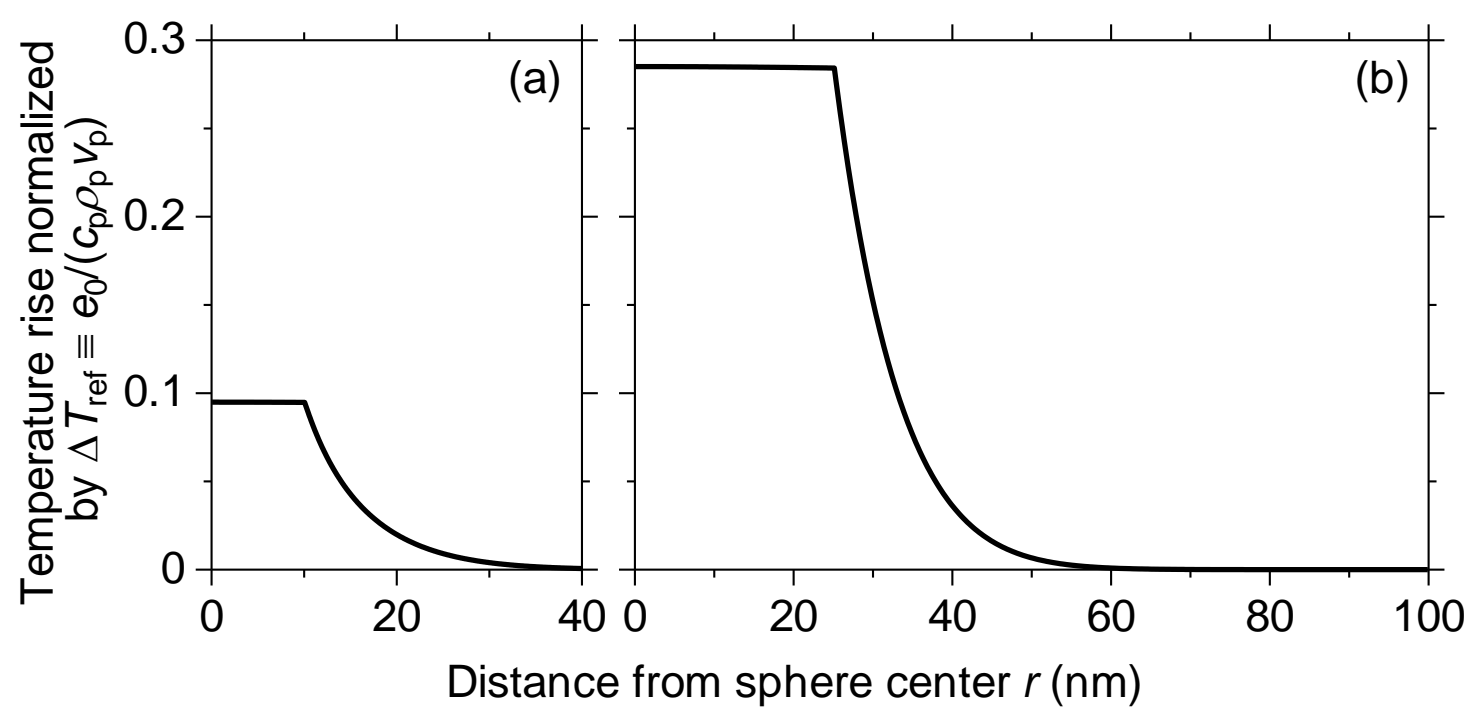

Fig. 4. The spatial distribution of the temperature rise, $\Delta T(r, t)$, around a spherical gold NP in water at $20{ }^{\circ} \mathrm{C}$ after $t=0.8 \mathrm{~ns}$ : (a) $d_{\mathrm{p}}=20 \mathrm{~nm}$ and (b) $d_{\mathrm{p}}=50 \mathrm{~nm}$. The temperature rise was normalized by the maximum temperature rise of the particle without heat loss $\Delta T_{\text {ref }} \equiv e_{0} /\left(c_{\mathrm{p}} \rho_{\mathrm{p}} v_{\mathrm{p}}\right)$, where $e_{0} \equiv \sigma_{\mathrm{abs}} F_{0}$ represents the optical power deposition per particle. Almost the same simulation results were obtained for $4{ }^{\circ} \mathrm{C}$.

efficiencies of the spherical gold NPs in water were calculated following Ref. [24], where $Q_{\text {abs }} \equiv$ $\sigma_{\mathrm{abs}} / \pi r_{\mathrm{p}}{ }^{2}=1.12$ and 3.17 at $\lambda=522 \mathrm{~nm}$ were obtained for $d_{\mathrm{p}}=20$ and $50 \mathrm{~nm}$, respectively. It should be noted that we implicitly assumed no thermal expansion during/after laser irradiation. Figure 4 shows the spatial distribution of the temperature rise around the gold NPs at $20{ }^{\circ} \mathrm{C}$ after $t=0.8 \mathrm{~ns}(=$ $t_{\mathrm{L}}$, immediately after the laser irradiation), when the temperature of the NPs became maximal. The temperature rise was normalized by $\Delta T_{\text {ref }} \equiv e_{0} /\left(c_{\mathrm{p}} \rho_{\mathrm{p}} v_{\mathrm{p}}\right)$, where $e_{0} \equiv \sigma_{\mathrm{abs}} F_{0}$ represents the optical power deposition per NP. As seen from Fig. 4, the temperatures inside the NP $\left(r \leq r_{\mathrm{p}}\right)$ were almost homogeneous regardless of the NP diameters considered in the present study $\left(d_{\mathrm{p}} \leq 60 \mathrm{~nm}\right)$. This is due to the large thermal diffusivity of gold, $\chi_{\mathrm{p}}\left(\equiv k_{\mathrm{p}} / c_{\mathrm{p}} \rho_{\mathrm{p}}\right)=1.27 \times 10^{-4} \mathrm{~m}^{2} / \mathrm{s}$. The thermal diffusion distance of gold for the laser pulse duration is calculated as $\left(\chi_{\mathrm{p}} t_{\mathrm{L}}\right)^{1 / 2}=319 \mathrm{~nm}$, which is about ten times larger than the radii of NPs considered in the present study ( $r_{\mathrm{p}} \leq 30 \mathrm{~nm}$ ). A comparison between parts $a$ and $b$ of Fig. 4 indicates that the temperature inside the NP increased with the NP diameter. The heated volumes were highly localized to the vicinity of the NPs, although there was 


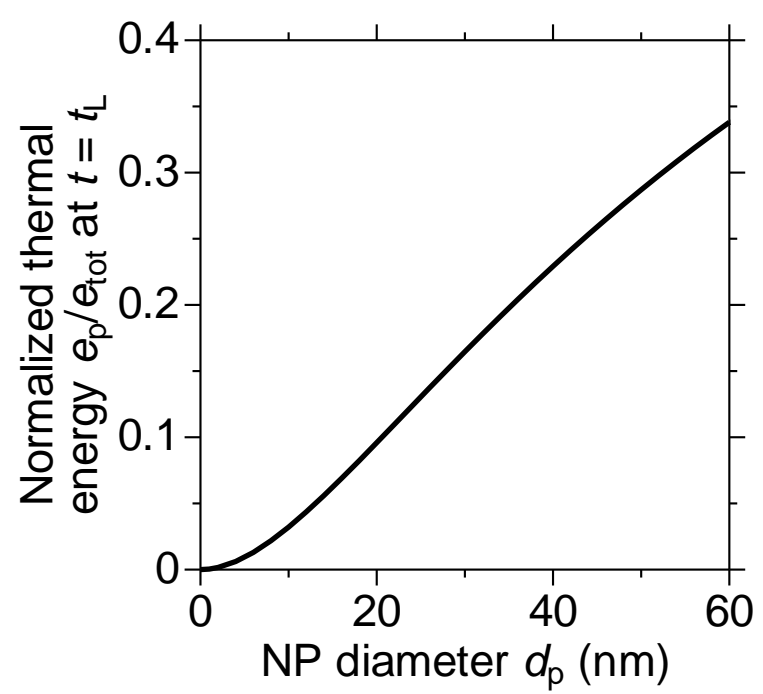

Fig. 5. The ratio between the thermal energy inside the NP and the total thermal energy, $e_{\mathrm{p}} / e_{\mathrm{tot}}$, for a spherical gold NP in water at $20{ }^{\circ} \mathrm{C}$ after $t=t_{\mathrm{L}}=0.8 \mathrm{~ns}$, as a function of the NP diameter. Almost the same simulation result was obtained for $4{ }^{\circ} \mathrm{C}$.

Figure 5 shows the ratio between the thermal energy inside the NP and the total thermal energy, $e_{\mathrm{p}} / e_{\mathrm{tot}}$ for a spherical gold NP in water at $20^{\circ} \mathrm{C}$ after $t=t_{\mathrm{L}}=0.8 \mathrm{~ns}$, as a function of the NP

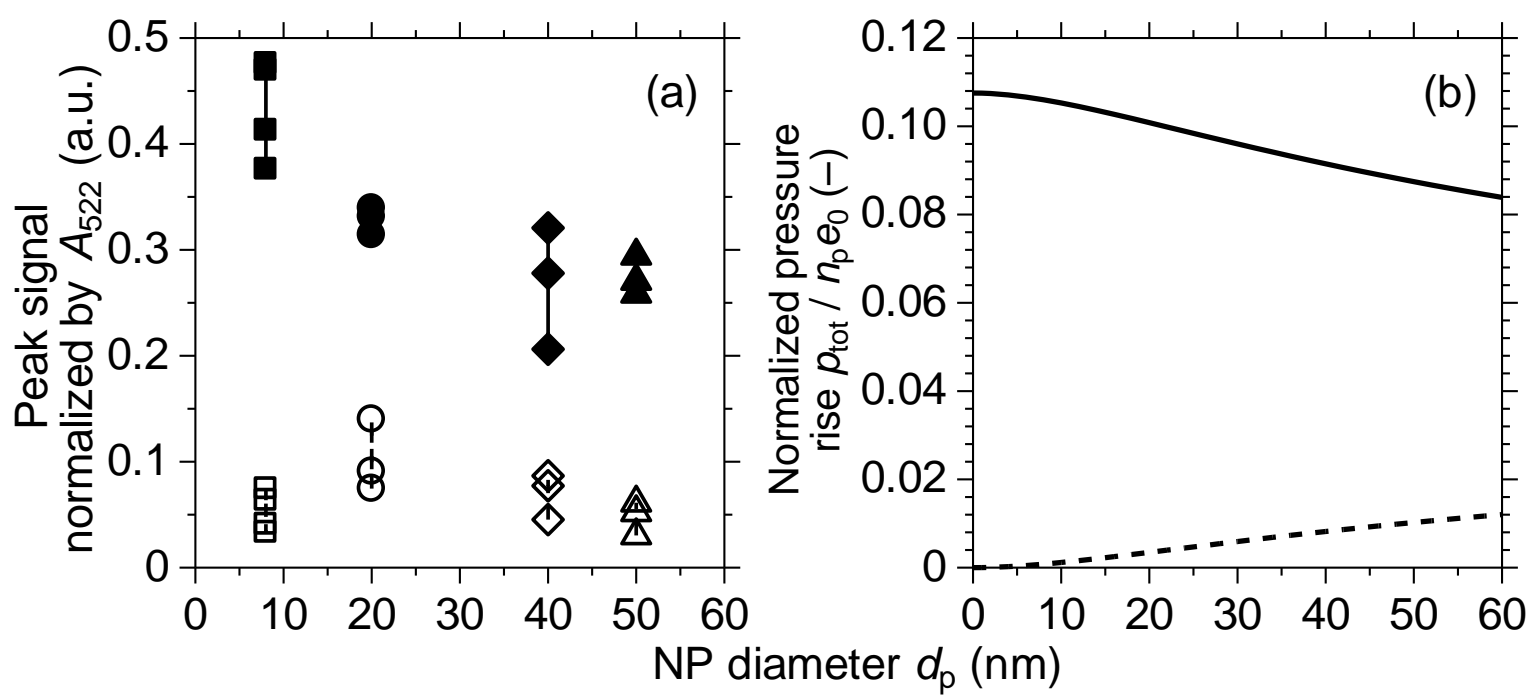

Fig. 6. (a) Replot of the experimental data in Fig. 3 as a function of the NP diameter for different temperatures of $4^{\circ} \mathrm{C}$ (open symbols) and $20^{\circ} \mathrm{C}$ (filled symbols), where every peak value of the photoacoustic signal was normalized by the absorbance of the NP suspension $A_{522}$. The results of $d_{\mathrm{p}}=8 \mathrm{~nm}$ (squares) taken from Ref. [19] are also shown. (b) The acoustic pressure pulse normalized by the optical power deposition of the suspension per unit volume for the systems corresponding to panel a at $4{ }^{\circ} \mathrm{C}$ (dashed line) and $20^{\circ} \mathrm{C}$ (solid line). 


\subsection{Comparison between experimental results and theoretical estimations}

As shown in Fig. 6a, the experimental data in Fig. 3 is replotted as a function of the NP diameter, where every peak value of the photoacoustic signal was normalized by the absorbance of the NP suspension $A_{522}$. The results of $d_{\mathrm{p}}=8 \mathrm{~nm}$ taken from our previous study [19] are also shown in Fig. 6a. According to Eq. (14), the photoacoustic signal normalized by the absorbance $\left(p_{\text {tot }} / A_{\lambda}\right)$ should be constant for a given NP diameter $\left(d_{\mathrm{p}}\right)$ regardless of the absorbance $\left(A_{\lambda}\right)$ or the NP concentration $\left(n_{\mathrm{p}}\right)$. Indeed, this is the case for Fig. 6a, though some scatters are observed: this result demonstrates the accuracy of our experimental observation as well as the validity of our phenomenological modeling.

In the case of $20^{\circ} \mathrm{C}$, the normalized peak signal decreased with increasing $d_{\mathrm{p}}$. This result suggests that even when the total energy of an NP suspension per unit volume produced by the laser irradiation is constant, the resultant photoacoustic pressure is greatly influenced by the NP size. In the case of $4{ }^{\circ} \mathrm{C}$, the normalized peak signals were significantly reduced compared with those at $20{ }^{\circ} \mathrm{C}$.

Figure $6 \mathrm{~b}$ displays the calculated acoustic pressure pulse $\left(p_{\text {tot }}\right)$ as a function of $d_{\mathrm{p}}$, where every $p_{\text {tot }}$ was normalized by $n_{\mathrm{p}} e_{0}$ that represents the optical power deposition of the suspension per unit volume. In the case of $20{ }^{\circ} \mathrm{C}$, the predicted pressure pulse decreased with increasing $d_{\mathrm{p}}$; this behavior was in good agreement with the experimental result shown in Fig. 6a. The dimensionless prefactors for the NP and medium contributions in Eq. (1) were calculated as $\kappa_{\mathrm{p}} \Gamma_{\mathrm{p}} / \kappa_{\mathrm{m}}=0.038$ and $\Gamma_{\mathrm{m}}=0.108$, respectively. Using these values, Eq. (1) or (14) with $e_{\text {tot }}=e_{0}$ can be rewritten as $p_{\text {tot }} / n_{\mathrm{p}} e_{0}$ $=-0.070 x+0.108$, where $x$ monotonically increases from zero to unity with increasing $d_{\mathrm{p}}$ (see Fig. 5). The negative sign of the prefactor for $x$ in Eq. (14) explains the monotonic decrease of the normalized acoustic pressure with the NP size shown in Fig. 6b.

In the case of $4{ }^{\circ} \mathrm{C}$, the calculated acoustic pressure pulses were considerably diminished, where Eq. (1) is reduced to $p_{\text {tot }} / n_{\mathrm{p}} e_{0}=0.038 x$ because of $\beta_{\text {water }}=0$ at $4{ }^{\circ} \mathrm{C}$. The acoustic pressure pulse at 4 ${ }^{\circ} \mathrm{C}$ was never contributed by a great amount of the heat within the water medium (i.e., $e_{\mathrm{m}} / e_{\mathrm{tot}}=1-$ 
$e_{\mathrm{p}} / e_{\text {tot }}>0.66$ as in Fig. 5) because of $\beta_{\text {water }}=0$ as in Table S1, but solely by a negligibly small amount of the heat within the NP (i.e., $e_{\mathrm{p}} / e_{\mathrm{tot}}<0.34$ as in Fig. 5). This extremely low efficiency of the thermal-to-acoustic conversion at $4{ }^{\circ} \mathrm{C}$ resulted in the negligibly small values of acoustic pressure pulses compared with the case of $20^{\circ} \mathrm{C}$, as in Fig. $6 \mathrm{~b}$. It is noted that the pressure pulse predicted at $9{ }^{\circ} \mathrm{C}$ is constant regardless of the NP size (i.e, $p_{\text {tot }} / n_{\mathrm{p}} e_{0}=0.037$ ), where the temperaturedependent properties of water were used as listed in Table S2 and the physical properties of gold were assumed to be constant regardless of temperature as in Tale S1.

The simulation results in Fig. $6 \mathrm{~b}$ generally agreed with the experimental results in Fig. 6a, though there were some variances between them at $4{ }^{\circ} \mathrm{C}$. This would be explained by the following two reasons. First, it was technically difficult to measure with precision the photoacoustic signal amplitudes in water at $4{ }^{\circ} \mathrm{C}$ that were relatively small and close to the value of the background noise (see Fig. 3). Second, the assumptions made in our phenomenological modeling are arguable, where the physical properties of an NP and its surrounding medium were assumed to exhibit the constant values at the initial temperature during the laser irradiation; especially, the assumption of $\beta_{\text {water }}=0$ at $4{ }^{\circ} \mathrm{C}$ seems more or less premature. Although the temperature rise of the whole suspension is sufficiently small ( several $\mathrm{mK})$ during laser irradiation, that of the medium in the vicinity of an NP is large according to our estimation of the temperature profiles (see Fig. 4). This suggests that the contribution of acoustic pressure from the water medium is not negligible even at the temperature point of $4{ }^{\circ} \mathrm{C}$ [28]. Therefore, it is desirable to take this thermal nonlinearity into consideration, when modeling the photoacoustic phenomena at $4{ }^{\circ} \mathrm{C}[19]$. This will be our future study.

\section{Conclusions}

We carried out the experimental measurements of the photoacoustic responses, where the suspensions of spherical gold NPs of different diameters $(20,40$, and $50 \mathrm{~nm})$ in water at different concentrations and temperatures $\left(4\right.$ and $\left.20^{\circ} \mathrm{C}\right)$ were irradiated by nanosecond laser pulses. In the case of $20{ }^{\circ} \mathrm{C}$, the values of photoacoustic signals normalized by the light absorbance of the NP suspension decreased with increasing the NP size. This size-dependent behavior of the photoacoustic 
responses agreed with the prediction from our phenomenological model, where the acoustic pressure pulse is represented by a sum of two contributions from the NPs and the medium. In the case of $4{ }^{\circ} \mathrm{C}$, the photoacoustic signals were significantly reduced compared with those at $20{ }^{\circ} \mathrm{C}$. This result was reasonably explained by our model, though there were some variances between the experimental results and the theoretical estimations at $4{ }^{\circ} \mathrm{C}$. Further experimental and theoretical studies are required for understanding clearly how the magnitudes of photoacoustic responses from aqueous suspensions of gold NPs at $4{ }^{\circ} \mathrm{C}$ are influenced by the NP size.

\section{Acknowledgements}

This work was partly supported by the Innovative Techno-Hub for Integrated Medical Bioimaging of the Project for Developing Innovation Systems, from the Ministry of Education, Culture, Sports, Science and Technology (MEXT), Japan. 


\section{References}

[1] C. Li, L.V. Wang, Photoacoustic tomography and sensing in biomedicine, Phys. Med. Biol., 54 (2009) R59-R97.

[2] C. Kim, C. Favazza, L.V. Wang, In vivo photoacoustic tomography of chemicals: Highresolution functional and molecular optical imaging at new depths, Chem. Rev., 110 (2010) 27562782.

[3] C.M. Cobley, J. Chen, E.C. Cho, L.V. Wang, Y. Xia, Gold nanostructures: a class of multifunctional materials for biomedical applications, Chem. Soc. Rev., 40 (2011) 44-56.

[4] J. Yu, D. Javier, M.A. Yaseen, N. Nitin, R. Richards-Kortum, B. Anvari, M.S. Wong, Selfassembly synthesis, tumor cell targeting, and photothermal capabilities of antibody-coated indocyanine green nanocapsules, J. Am. Chem. Soc., 132 (2010) 1929-1938.

[5] M.A. Hahn, A.K. Singh, P. Sharma, S.C. Brown, B.M. Moudgil, Nanoparticles as contrast agents for in-vivo bioimaging: current status and future perspectives, Anal. Bioanal. Chem., 399 (2010) 3 27.

[6] X. Yang, E.W. Stein, S. Ashkenazi, L.V. Wang, Nanoparticles for photoacoustic imaging, WIREs Nanomedicine and Nanobiotechnology, 1 (2009) 360-368.

[7] S.S. Alimpiev, Y.O. Simanovskii, S.V. Egerev, A.E. Pashin, Optoacoustic detection of microparticles in liquids at laser fluences below the optical breakdown threshold, Laser Chem, 16 (1995) 63-73.

[8] C.P. Lin, M.W. Kelly, Cavitation and acoustic emission around laser-heated microparticles, Appl. Phys. Lett., 72 (1998) 2800-2802.

[9] S.V. Egerev, O.B. Ovchinnikov, A.V. Fokin, Optoacoustic conversion in suspensions: the competition of mechanisms and statistical characteristics, Acoust. Phys., 51 (2005) 160-166.

[10] S.V. Egerev, A.A. Oraevsky, Optothermoacoustic phenomena in highly diluted suspensions of gold nanoparticles, Int. J. Thermophys., 29 (2008) 2116-2125.

[11] S. Egerev, S. Ermilov, O. Ovchinnikov, A. Fokin, D. Guzatov, V. Kilmov, A. Kanavin, A. Oraevsky, Acoustic signals generated by laser-irradiated metal nanoparticles, Appl. Optics, 48 (2009) C38-C45. 
[12] V.P. Zharov, V. Galitovsky, M. Viegas, Photothermal detection of local thermal effects during selective nanophotothermolysis, Appl. Phys. Lett., 83 (2003) 4897-4899.

[13] V. Kotaidis, A. Plech, Cavitation dynamics on the nanoscale, Appl. Phys. Lett., 87 (2005) 213102.

[14] S.R. Aglyamov, A.B. Karpiouk, F. Bourgeois, A. Ben-Yakar, S.Y. Emelianov, Ultrasound measurements of cavitation bubble radius for femtosecond laser-induced breakdown in water, Optics Letters, 33 (2008) 1357-1359.

[15] M.G. González, X. Liu, R. Niessner, C. Haisch, Strong size-dependent photoacoustic effect on gold nanoparticles by laser-induced nanobubbles, Appl. Phys. Lett., 96 (2010) 174104.

[16] M. Kitz, S. Preisser, A. Wetterwald, M. Jaeger, G.N. Thalmann, M. Frenz, Vapor bubble generation around gold nanoparticles and its application to damaging of cells, Biomed. Opt. Express, 2 (2011) 291-304.

[17] E.A. Brujan, Numerical investigation on the dynamics of cavitation nanobubbles, Microfluid. Nanofluid., 11 (2011) 511-517.

[18] Y.S. Chen, W. Frey, S. Aglyamov, S. Emelianov, Environment-dependent generation of photoacoustic waves from plasmonic nanoparticles, Small, 8 (2012) 47-52.

[19] H. Shinto, T. Fukasawa, H. Aoki, S. Ito, M. Ohshima, Acoustic pressure pulses from laserirradiated suspensions containing gold nanospheres in water: Experimental and theoretical study, Colloids. Surf. A, 430 (2013) 51-57.

[20] O.M. Zozulya, O.V. Puchenkov, On theory of optoacoustic effect in liquid disperse systems, Acoust. Phys., 39 (1993) 46-50.

[21] V.N. Inkov, A.A. Karabutov, I.M. Pelivanov, A theoretical model of the linear thermo-optical response of an absorbing particle immersed in a liquid, Laser Phys., 11 (2001) 1283-1291.

[22] Y.N. Cao, G.J. Diebold, Effects of heat conduction and viscosity on photoacoustic waves from droplets, Opt. Eng., 36 (1997) 417-422.

[23] L.V. Wang, Tutorial on photoacoustic microscopy and computed tomography, IEEE J. Sel. Top. Quant. Electron., 14 (2008) 171-179. 
[24] P.K. Jain, K.S. Lee, I.H. El-Sayed, M.A. El-Sayed, Calculated absorption and scattering properties of gold nanoparticles of different size, shape, and composition: applications in biological imaging and biomedicine, J. Phys. Chem. B, 110 (2006) 7238-7248.

[25] S.V. Patankar, Numerical Heat Transfer and Fluid Flow, Taylor \& Francis, 1980.

[26] J. Copland, M. Eghtedari, V. Popov, N. Kotov, N. Mamedova, M. Motamedi, A. Oraevsky, Bioconjugated gold nanoparticles as a molecular based contrast agent: implications for imaging of deep tumors using optoacoustic tomography, Mol. Imaging Biol., 6 (2004) 341-349.

[27] S.V. Egerev, A.A. Oraevsky, Nonlinear optothermoacoustic phenomena in highly diluted suspensions of gold nanoparticles, J.Phys. IV France, 137 (2006) 273-281.

[28] M.W. Sigrist, Laser generation of acoustic-waves in liquids and gases, J. Appl. Phys., 60 (1986) R83-R121. 


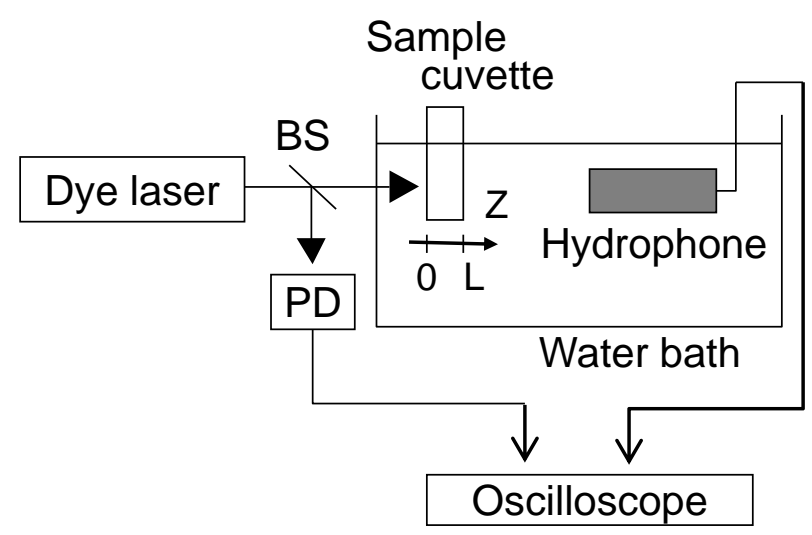

Fig. 1 Fukasawa et al. 


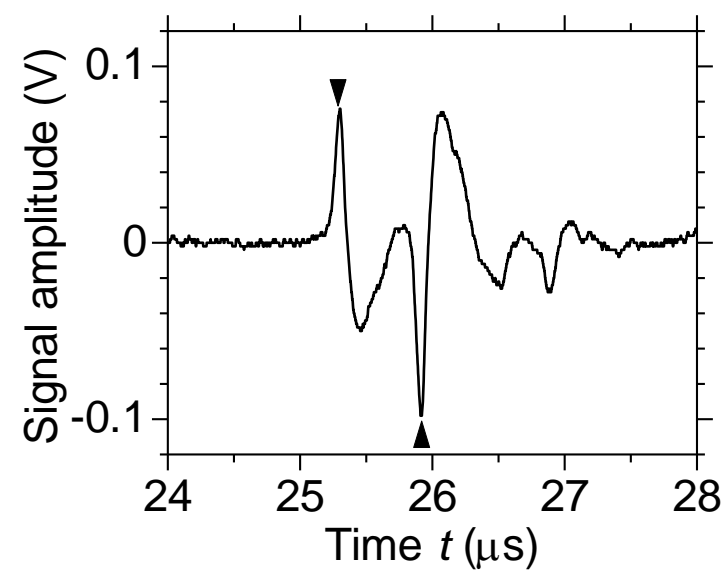

Fig. 2 Fukasawa et al. 


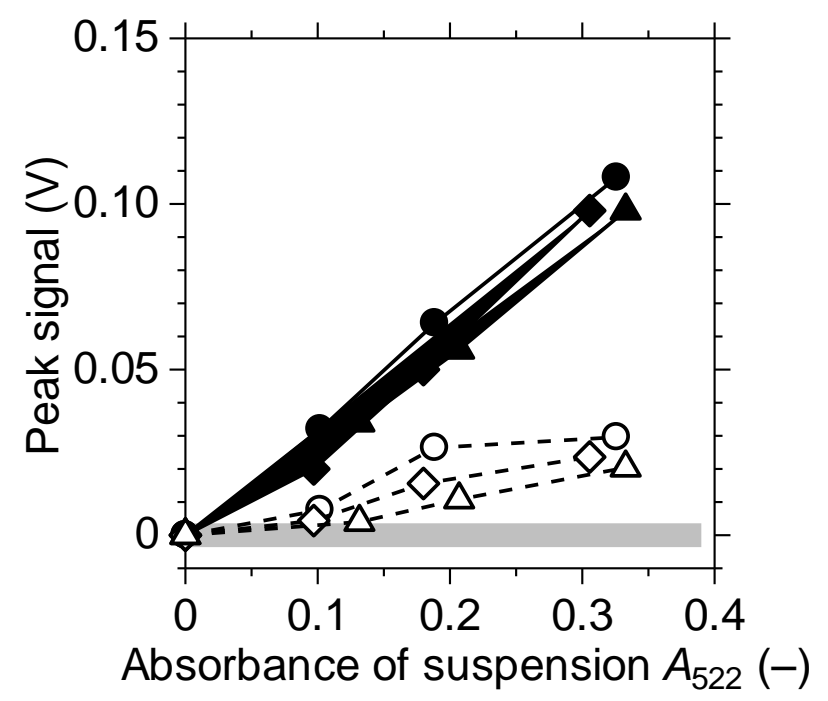

Fig. 3 Fukasawa et al. 


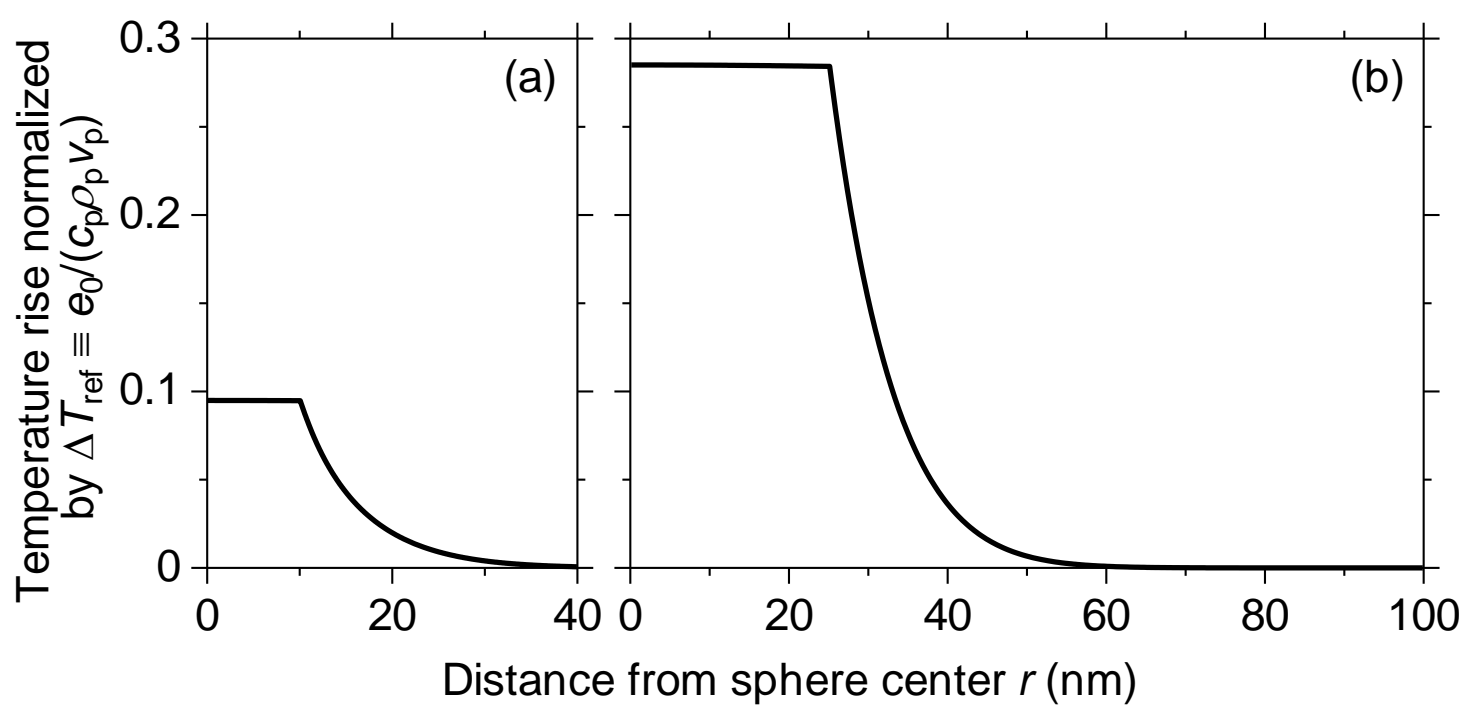

Fig. 4 Fukasawa et al. 


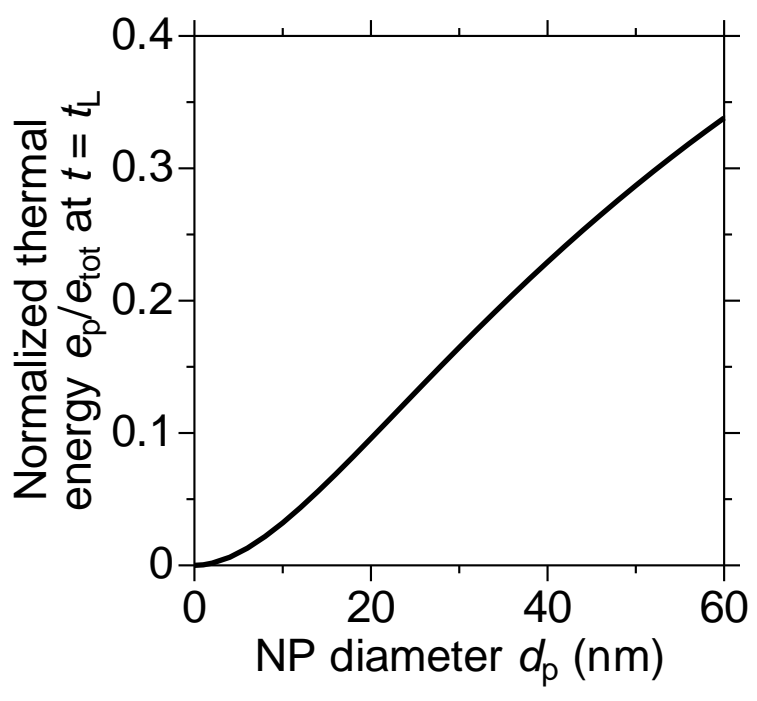

Fig. 5 Fukasawa et al. 


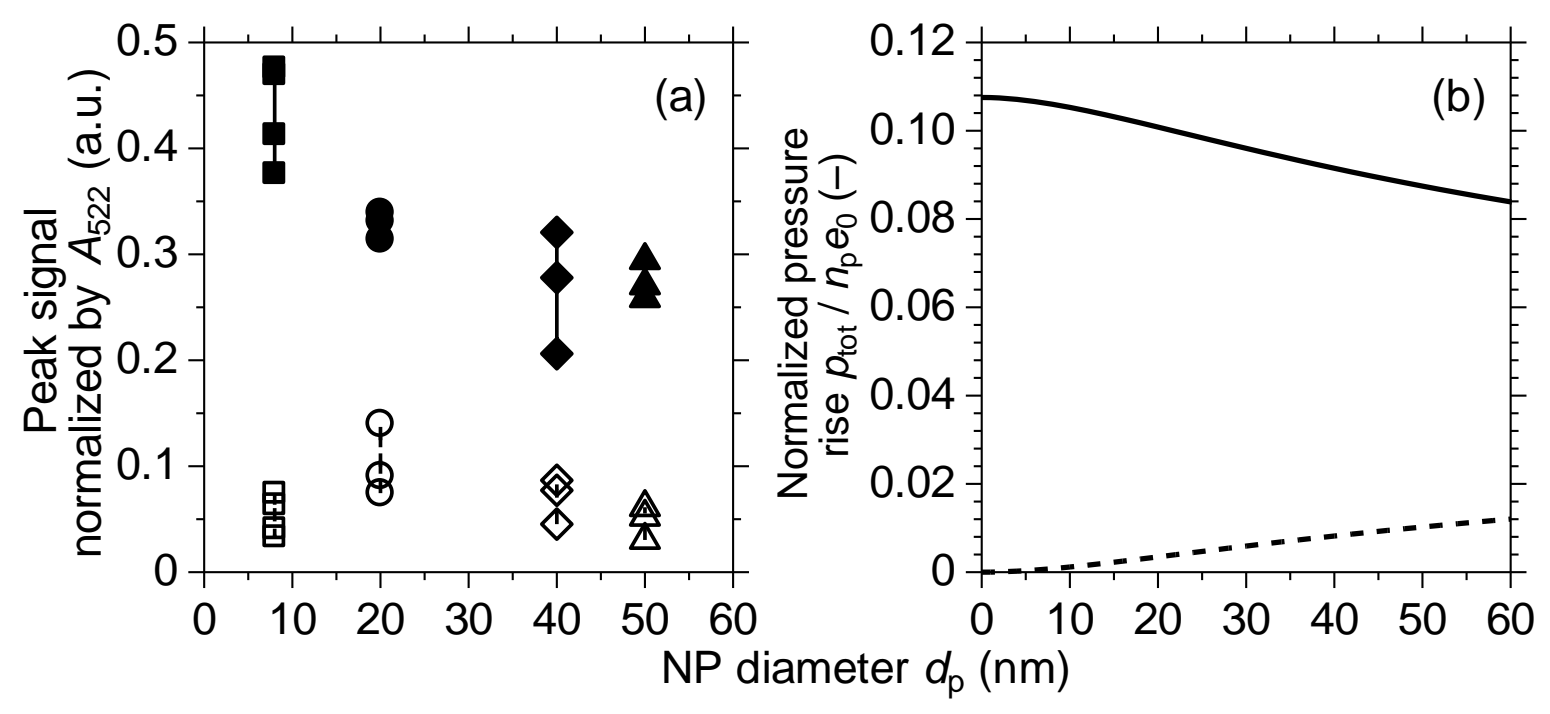

Fig. 6 Fukasawa et al. 


\section{Electronic Supplementary Data}

\section{Size-dependent effect of gold nanospheres on the acoustic pressure pulses from laser- irradiated suspensions}

Tomonori Fukasawa $^{1}$, Hiroyuki Shinto ${ }^{1, *}$, Hiroyuki Aoki ${ }^{2,3}$, Shinzaburo Ito $^{2,3}$, and Masahiro Ohshima ${ }^{1}$

${ }^{1}$ Department of Chemical Engineering, ${ }^{2}$ Department of Polymer Chemistry, and ${ }^{3}$ Advanced Biomedical Engineering Research Unit, Kyoto University, Katsura, Nishikyo-ku, Kyoto 615-8510, Japan

*Author to whom correspondence should be addressed.

Phone: +81-75-383-2671, Fax: +81-75-383-2651, e-mail: shinto@ cheme.kyoto-u.ac.jp 

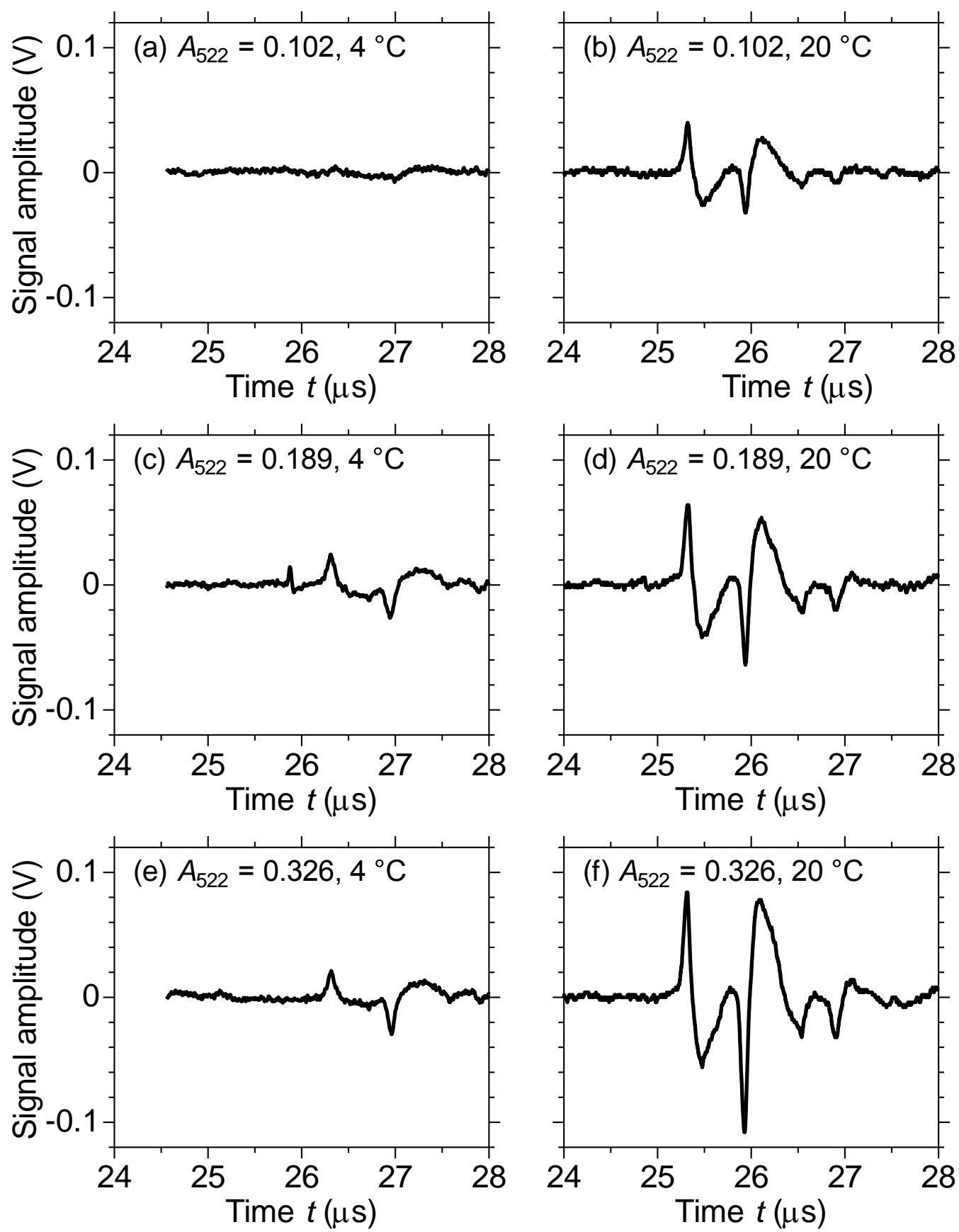

Fig. S1. Time variation of signal amplitudes observed from a laser-irradiated aqueous suspension of 20-nm gold NPs. $A_{522}$ represents the observed absorbance of the suspension through the cuvette of the light pass length of $L=0.92 \mathrm{~mm}$ at a wavelength of $522 \mathrm{~nm}$. (a) $A_{522}=0.102,4{ }^{\circ} \mathrm{C}$; (b) $A_{522}=0.102$, $20{ }^{\circ} \mathrm{C}$; (c) $A_{522}=0.189,4{ }^{\circ} \mathrm{C}$; (d) $A_{522}=0.189,20{ }^{\circ} \mathrm{C}$; (e) $A_{522}=0.326,4{ }^{\circ} \mathrm{C}$; and (f) $A_{522}=0.326$, $20{ }^{\circ} \mathrm{C}$. 

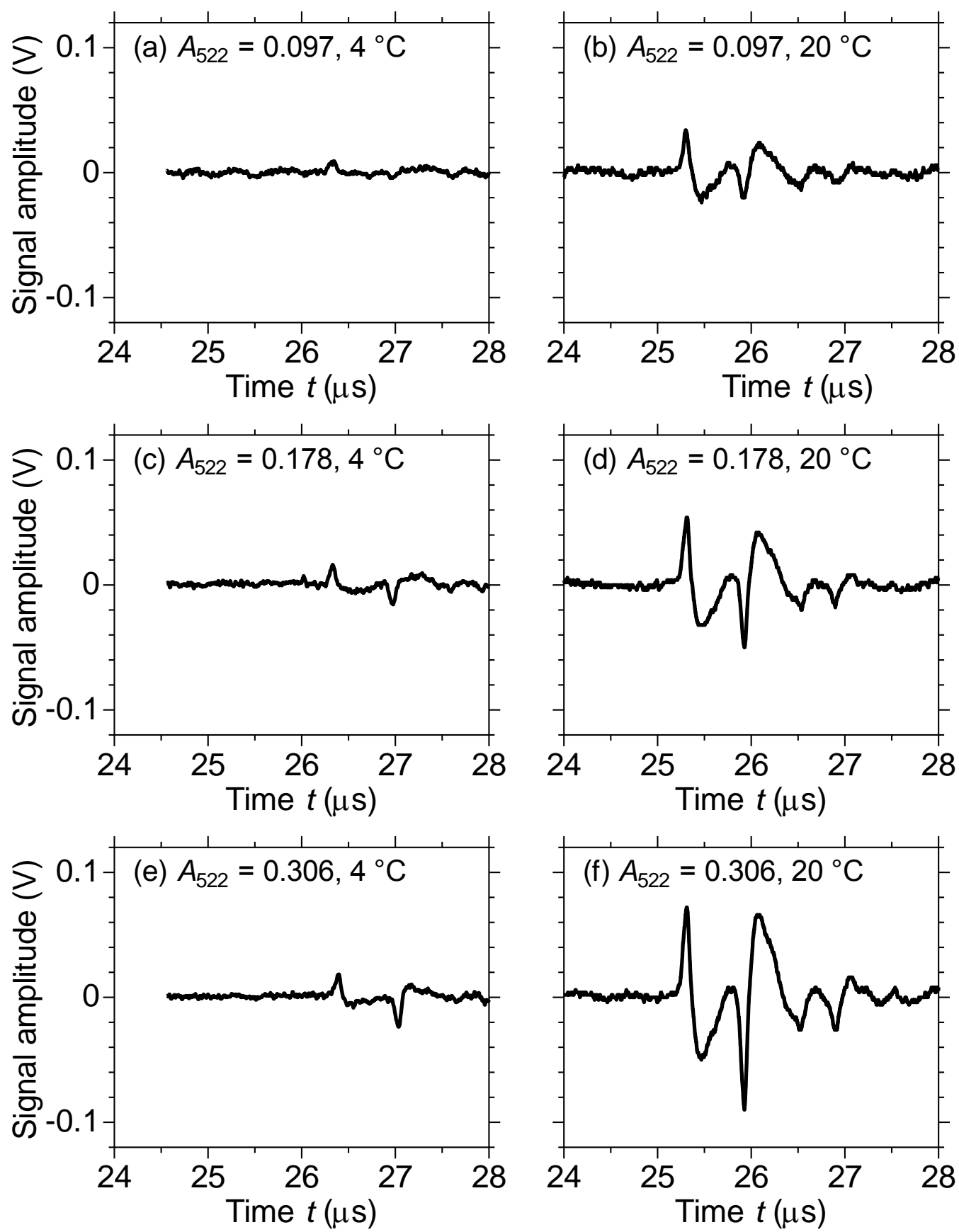

Fig. S2. Time variation of signal amplitudes observed from a laser-irradiated aqueous suspension of 40-nm gold NPs. $A_{522}$ represents the observed absorbance of the suspension through the cuvette of the light pass length of $L=0.92 \mathrm{~mm}$ at a wavelength of $522 \mathrm{~nm}$. (a) $A_{522}=0.097,4{ }^{\circ} \mathrm{C}$; (b) $A_{522}=0.097$, $20{ }^{\circ} \mathrm{C}$; (c) $A_{522}=0.178,4{ }^{\circ} \mathrm{C}$; (d) $A_{522}=0.178,20{ }^{\circ} \mathrm{C}$; (e) $A_{522}=0.306,4{ }^{\circ} \mathrm{C}$; and (f) $A_{522}=0.306$, $20{ }^{\circ} \mathrm{C}$. 

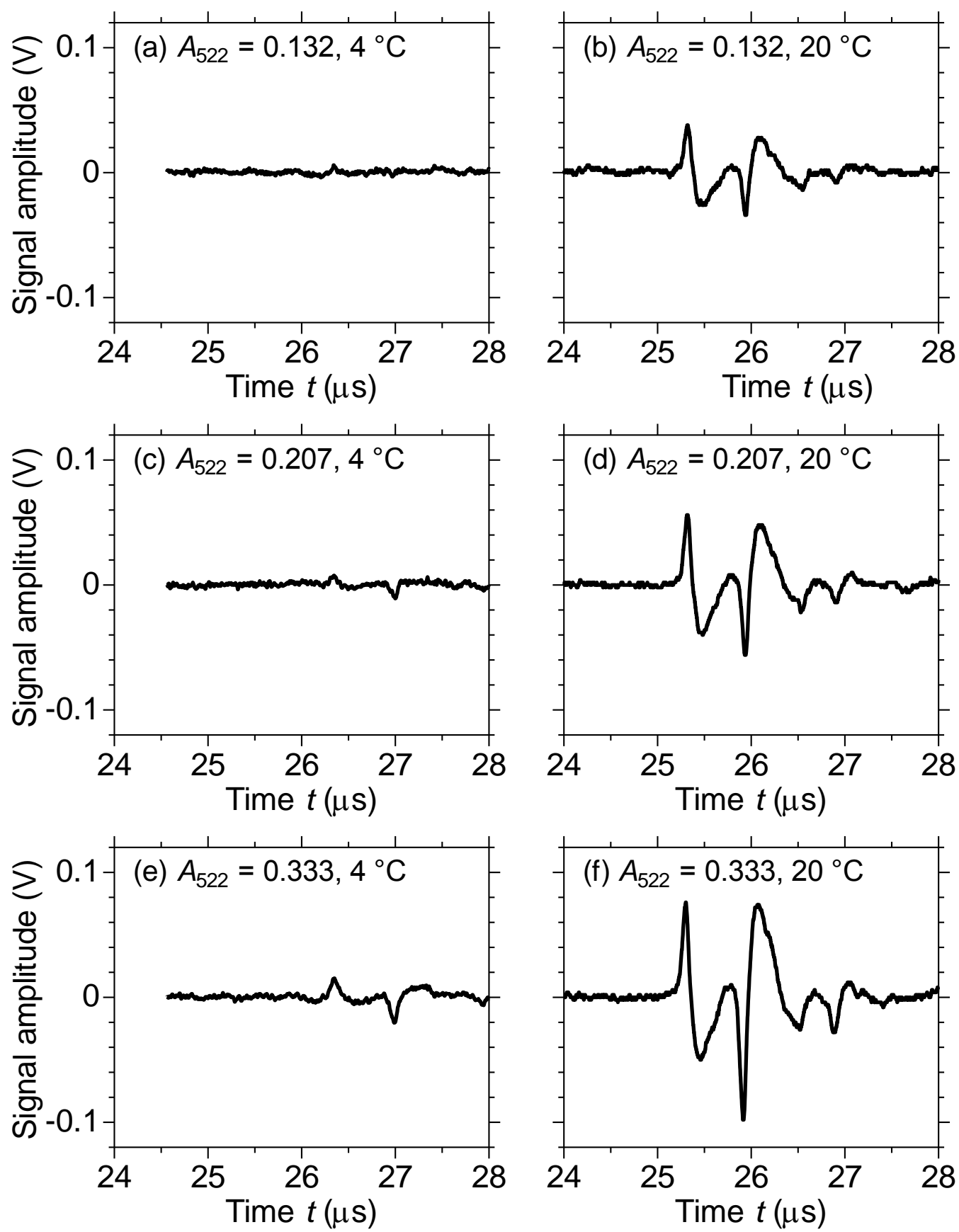

Fig. S3. Time variation of signal amplitudes observed from a laser-irradiated aqueous suspension of 50-nm gold NPs. $A_{522}$ represents the observed absorbance of the suspension through the cuvette of the light pass length of $L=0.92 \mathrm{~mm}$ at a wavelength of $522 \mathrm{~nm}$. (a) $A_{522}=0.132,4{ }^{\circ} \mathrm{C}$; (b) $A_{522}=0.132$, $20{ }^{\circ} \mathrm{C}$; (c) $A_{522}=0.207,4{ }^{\circ} \mathrm{C}$; (d) $A_{522}=0.207,20{ }^{\circ} \mathrm{C}$; (e) $A_{522}=0.333,4{ }^{\circ} \mathrm{C}$; and (f) $A_{522}=0.333$, $20{ }^{\circ} \mathrm{C}$. 


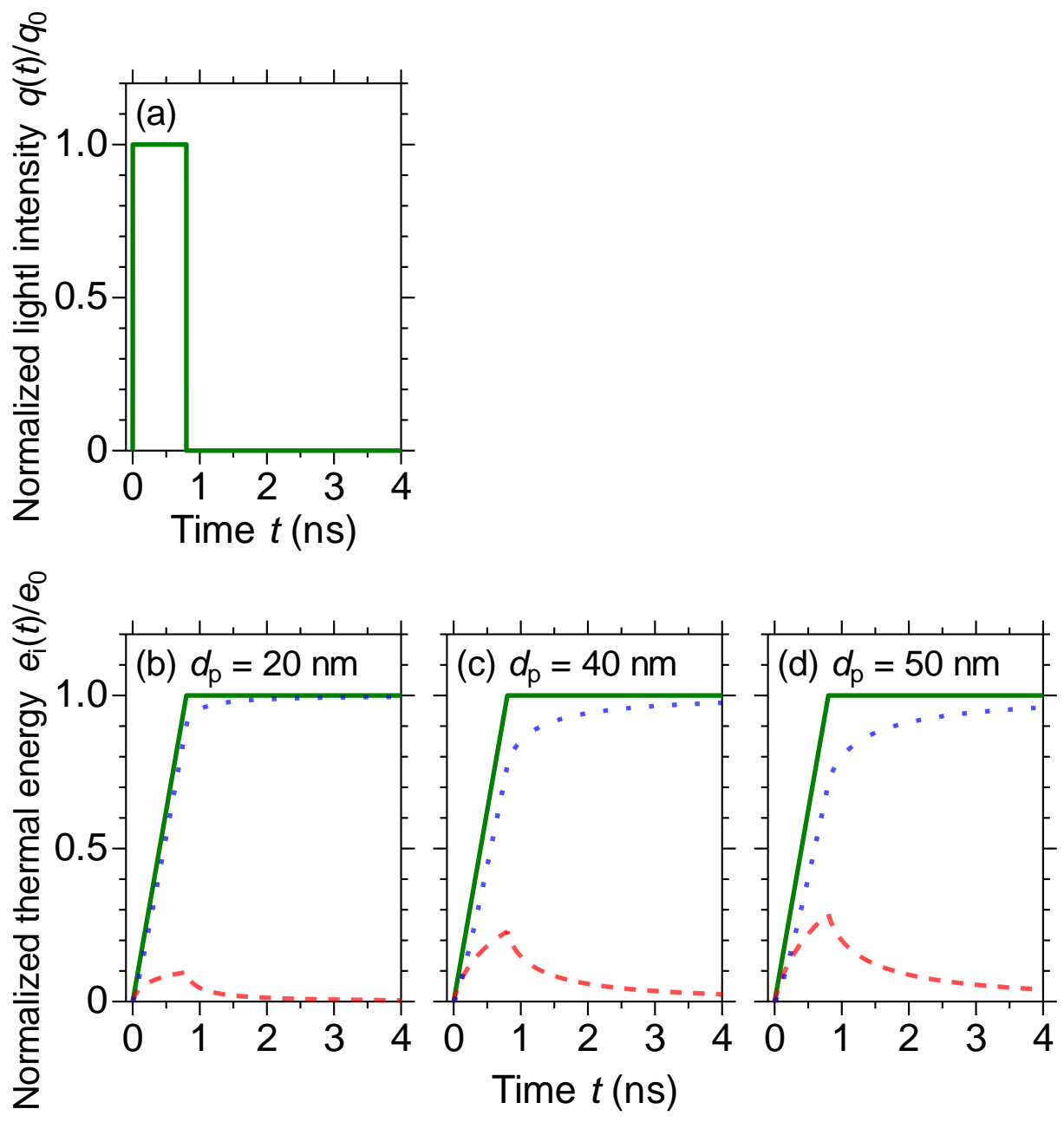

Fig. S4. (a) The normalized intensity of a laser beam with a rectangular pulse of FWHM $t_{\mathrm{L}}=0.8 \mathrm{~ns}$, as a function of time $t$. (b,c,d) The total thermal energy produced by a laser-irradiated gold NP of three different diameters $\left[e_{\mathrm{tot}}(t)\right.$, solid line, green $]$, the thermal energy inside the NP $\left[e_{\mathrm{p}}(t)\right.$, long dashed line, red], and the thermal energy of its surrounding water medium $\left[e_{\mathrm{m}}(t)\right.$, short dashed line, blue $]$, as a function of time $t$ at $20^{\circ} \mathrm{C}$ : (b) $d_{\mathrm{p}}=20 \mathrm{~nm},(\mathrm{c}) d_{\mathrm{p}}=40 \mathrm{~nm}$, and (d) $d_{\mathrm{p}}=50 \mathrm{~nm}$. Every energy $e_{\mathrm{i}}(t)(\mathrm{i}=$ tot, $\mathrm{p}, \mathrm{m}$ ) was normalized by $e_{0}$, which represents the optical power deposition per NP. 
Table S1. Physical properties of gold (particle) and water (medium) at ambient conditions (taken from Ref. [19]).

\begin{tabular}{lccc}
\hline Physical property & Gold & $\begin{array}{c}\text { Water } \\
\text { at } 4^{\circ} \mathrm{C}\end{array}$ & $\begin{array}{c}\text { Water } \\
\text { at } 20{ }^{\circ} \mathrm{C}\end{array}$ \\
\hline$\rho_{\mathrm{i}}:$ Mass density $\left(\mathrm{kg} \mathrm{m}^{-3}\right)$ & 19320 & 1000 & 998 \\
$c_{\mathrm{i}}:$ Specific heat capacity $\left(\mathrm{J} \mathrm{kg}^{-1} \mathrm{~K}^{-1}\right)$ & 128 & 4205 & 4182 \\
$k_{\mathrm{i}}$ : Thermal conductivity $\left(\mathrm{J} \mathrm{K}^{-1} \mathrm{~m}^{-1} \mathrm{~s}^{-1}\right)$ & $(315)^{\mathrm{a}}$ & 0.567 & 0.599 \\
$\chi_{\mathrm{i}}$ : Thermal diffusivity $\left(\mathrm{m}^{2} \mathrm{~s}^{-1}\right)$ & $\left(1.27 \times 10^{-4}\right)^{\mathrm{a}}$ & $1.35 \times 10^{-7}$ & $1.43 \times 10^{-7}$ \\
$\beta_{\mathrm{i}}$ : Thermal coefficient of volume expansion $\left(\mathrm{K}^{-1}\right)$ & $4.27 \times 10^{-5}$ & 0 & $2.06 \times 10^{-4}$ \\
$\kappa_{\mathrm{i}}:$ Isothermal compressibility $\left(\mathrm{Pa}^{-1}\right)$ & $5.88 \times 10^{-12}$ & $4.96 \times 10^{-10}$ & $4.59 \times 10^{-10}$ \\
$\Gamma_{\mathrm{i}}=\beta_{\mathrm{i}} / \kappa_{\mathrm{i}} c_{\mathrm{i}} \rho_{\mathrm{i}}$ : Grüneisen parameter $(-)$ & 2.94 & 0 & 0.108 \\
Dimensionless prefactor for $p_{\mathrm{i}}$ in Eqs. $(1)$ or $(14)$ & $0.038^{\mathrm{b}}$ & $0^{\mathrm{c}}$ & $0.108^{\mathrm{c}}$ \\
\hline
\end{tabular}

\footnotetext{
${ }^{\mathrm{a}}$ The value in the parenthesis was not used in the solutions of the heat transfer equations.

${ }^{\mathrm{b}}$ The value of the prefactor $\kappa_{\mathrm{p}} \Gamma_{\mathrm{p}} / \kappa_{\mathrm{m}}$ for the particle contribution in Eqs. (1) or (14).

${ }^{\mathrm{c}}$ The value of the prefactor $\Gamma_{\mathrm{m}}$ for the medium contribution in Eqs. (1) or (14).
} 
Table S2. Coefficients $a_{i}(i=0,1,2,3$, and 4$)$ of predictive equations for physical properties of water (medium) at different temperature $T: f\left(T /{ }^{\circ} \mathrm{C}\right)=a_{0}+a_{1} T+a_{2} T^{2}+a_{3} T^{3}+a_{4} T^{4}$.

\begin{tabular}{lccccc}
\hline \multicolumn{1}{c}{ Physical property } & $a_{0}$ & $a_{1}$ & $a_{2}$ & $a_{3}$ & $a_{4}$ \\
\hline$\rho_{\mathrm{m}}:$ Mass density $\left(\mathrm{kg} \mathrm{m}^{-3}\right)^{\mathrm{a}}$ & $1.00 \times 10^{3}$ & $1.97 \times 10^{-2}$ & $-5.94 \times 10^{-3}$ & $1.59 \times 10^{-5}$ & \\
$c_{\mathrm{m}}$ : Specific heat capacity $\left(\mathrm{J} \mathrm{kg}^{-1} \mathrm{~K}^{-1}\right)^{\mathrm{b}}$ & $4.22 \times 10^{3}$ & -3.71 & 0.138 & $-2.5 \times 10^{-3}$ & $2.0 \times 10^{-5}$ \\
$\beta_{\mathrm{m}}:$ Thermal coefficient of volume & & & & & \\
expansion $\left(10^{-5} \mathrm{~K}^{-1}\right)^{\mathrm{c}}$ & -6.93 & 1.77 & $-2.33 \times 10^{-2}$ & $2.24 \times 10^{-4}$ & $-8.61 \times 10^{-4}$ \\
$\kappa_{\mathrm{m}}:$ Isothermal compressibility & & & & & \\
$\left(10^{-10} \mathrm{~Pa}^{-1}\right)^{\mathrm{a}}$ & 5.10 & $-3.69 \times 10^{-2}$ & $6.71 \times 10^{-4}$ & $-4.99 \times 10^{-6}$ & $1.75 \times 10^{-8}$ \\
\hline
\end{tabular}

$$
\begin{gathered}
{ }^{\mathrm{a}} 0 \leq T /{ }^{\circ} \mathrm{C} \leq 100 . \\
{ }^{\mathrm{b}} 0 \leq T /{ }^{\circ} \mathrm{C} \leq 40 . \\
{ }^{\mathrm{c}} 0 \leq T /{ }^{\circ} \mathrm{C} \leq 90 .
\end{gathered}
$$

